\title{
Association between retinol-binding protein 4 concentrations and gestational diabetes mellitus (A1GDM and A2GDM) in different pregnancy and postpartum periods
}

\author{
Xiyu Du ${ }^{1}$, Yunan Dong ${ }^{1}$, Li Xiao ${ }^{1}$, Guang-Hui Liu ${ }^{2}$, Wei Qin ${ }^{1}$, Hong Yu ${ }^{1}$ \\ ${ }^{1}$ Department of Obstetrics, Shenzhen maternal and child health care hospital affiliated to Southern Medical University, Shenzhen 518000, China; \\ ${ }^{2}$ Department of Endocrinology, Tongji Hospital affiliated to School of Medicine, Tongji University, Shanghai 200065, China \\ Contributions: (I) Conception and design: X Du; (II) Administrative support: X Du; (III) Provision of study materials or patients: X Du, Y Dong, L \\ Xiao, H Yu; (IV) Collection and assembly of data: X Du, Y Dong, L Xiao; (V) Data analysis and interpretation: X Du, GH Liu; (VI) Manuscript \\ writing: All authors; (VII) Final approval of manuscript: All authors. \\ Correspondence to: Guang-Hui Liu. Department of Endocrinology, Tongji Hospital affiliated to School of Medicine, Tongji University, Shanghai \\ 200065, China. Email: lghdoctor@tongji.edu.cn.
}

Background: Gestational diabetes mellitus (GDM) can cause severe adverse effects on fetal and neonatal outcomes. The following study investigates the relationship between retinol-binding protein 4 (RBP4) and GDM in pregnant women with different grades (A1 and A2) and different gestational weeks.

Methods: In this retrospective study, 194 GDM patients (GDM group) and 67 normal glucose tolerance pregnant women (control group) were enrolled from 2014 to 2017. Elbow venous blood samples were collected from all subjects. Enzyme electrode method and enzyme linked immunosorbent assay (ELISA) were used for fasting plasma glucose (FPG) and RBP4/insulin levels (FINS) analysis, respectively.

Results: At middle pregnancy and late stage, FINS, insulin resistance index (HOMA-IR) and RBP4 were all significantly higher in the GDM group compared to control group $(\mathrm{P}<0.05)$. Higher HOMA-IR and RBP4 levels, and lower levels of FPG were observed at late stage than those middle pregnancy in the GDM group $(\mathrm{P}<0.05)$. Moreover, FINS and RBP4 gradually decreased from middle pregnancy and late stage after delivery in the GDM group. Levels of FINS and RBP4 in postpartum GDM group were higher than those in normal control group $(\mathrm{P}<0.05)$. The optimal cut-off value of RBP4 at middle pregnancy diagnostic GDM was $34.84 \mu \mathrm{g} / \mathrm{mL}$ with sensitivity of $79.4 \%$ and specificity of $79.1 \%$. The OGTT0h, $2 \mathrm{~h}$ in A2GDM group was higher than that in A1GDM group, but there was no difference in OGTT1h, age and FINS, RBP4 in both A1GDM and A2GDM group.

Conclusions: RBP4 is closely related to GDM, and its levels increases with the increase of gestational weeks, which may reflect the development of insulin resistance in GDM. RBP4 suggests that the impaired insulin function of GDM in pregnant women is still difficult to recover in the short term after delivery. Compared with OGTT1h, the increase in OGTT0h and $2 \mathrm{~h}$ levels during middle pregnancy is more helpful for predicting the risk of developing A2GDM at late stage.

Keywords: Retinol-binding proteins; diabetes; gestational/gestational diabetes mellitus; insulin resistance

Submitted May 08, 2019. Accepted for publication Jul 24, 2019.

doi: $10.21037 /$ atm.2019.08.45

View this article at: http://dx.doi.org/10.21037/atm.2019.08.45 


\section{Introduction}

Gestational diabetes mellitus (GDM) is defined as any glucose intolerance first discovered or diagnosed during pregnancy, which can cause severe adverse effects on fetal and neonatal outcomes (1). Its prevalence ranges from $1 \%$ to $14 \%$ of all pregnancies worldwide (2). In Asia, the incidence of GDM is higher than $10 \%$ (3). GDM is adequately controlled with diet and exercise (A1GDM, also known as diet-controlled gestational diabetes) or medical therapy (A2GDM) (4). GDMA2 glucose metabolism disorder is more serious than A1GDM.

Besides, complications affecting mother and fetus are more frequent in GDM type 2 (5). Nevertheless, the exact mechanism of GDM occurrence is not clear. Relevant studies have found that the appearance of GDM may be associated with insulin resistance (IR) and relatively insufficient insulin secretion (6).

RBP4 is a protein which belongs to the lipocalin family. They are carrier proteins that bind to retinol. Increasing serum RBP4 induces hepatic expression of the gluconeogenic enzyme phosphoenolpyruvate carboxykinase (PEPCK) and impairs insulin signalling in muscle (7). RBP4 may have an important role in the origin of insulin resistance and metabolic syndrome (7-9). Some studies have suggested that RBP4 is closely related to type 2 diabetes (10). Feng et al. have found that RBP4 is positively correlated with T2DM and can be used as an early predictor of cardiovascular disease (11). Moreover, other studies have suggested an association between high RBP4 level and the development of GDM $(12,13)$. Contrary, other studies found no difference in RBP4 levels between GDM patients and normal pregnant women $(14,15)$.

Collecting samples and medical record for patients at middle pregnancy, late stage and after delivery can be very challenging. So far, only few studies were successful in tracking the results from pregnancy monitoring of the same women, and most of the available statistical data were obtained from different pregnant women, which is why these data might be inconsistent and biased. The current study lasted 3 years during which samples from more than 1,000 pregnant women were collected. By tracking the same pregnant woman at different gestational periods, the interference of GDM in pregnant women with obesity (16), age and other factors on RBP4 were also taken into account. There was no significant difference in age, gestational week and pre-pregnancy BMI among the subjects. Currently, there are not many research reports on the correlation between different grades (A1 and A2) at late stage of pregnancy obtained from the same GDM pregnant women. Consequently, we analyzed the RBP4 and relevant glucose metabolism biomarker levels in patients with A1GDM and A2GDM.

\section{Methods}

\section{Patients}

In this retrospective study, samples were collected from 1,000 patients diagnosed with GDM and no previous history of treatment (any medication given during middle pregnancy 24-28 weeks of pregnancy) at the obstetrics clinic of Shenzhen Maternal and Child Health Hospital affiliated to Southern Medical University from November 2014 to May 2017. Serum samples were taken from 1,000 GDM patients (considering the amount of subsequent patient loss to expand the specimen collection), and 300 healthy women (control group). Both groups were followed up to late stage (37 to 40 weeks of gestation). Finally, all pregnant women who have not been hospitalized at our obstetrics department for delivery or whose medical history data collection was incomplete or had other complications at late stage were excluded from the study. The inclusion criteria were the following: group objects were patients with monocyesis and no pregnancy complications such as hypertensive disorder, hyperthyroidism and other complications; nonsmokers, without the history of drug abuse, engaged in light physical work. The diagnosis of GDM was preformed using a singlestep 75-g OGTT when one or more of the following results were recorded during routine testing (specifically between 24 and 28 weeks of pregnancy or at any other time during the course of pregnancy): (I) fasting plasma glucose 5.1$6.9 \mathrm{mmol} / \mathrm{L}(92-125 \mathrm{mg} / \mathrm{dL})$; (II) 1 -hour post 75 -g oral glucose load $\geq 10 \mathrm{mmol} / \mathrm{L}$ (180 mg/dL); (III) 2 -hour post 75 -g oral glucose load 8.5-11.0 mmol/L (153-199 mg/dL) (17).

Finally, 194 patients with GDM (GDM group) and 67 pregnant women with normal glucose tolerance (control group) were enrolled; 108 GDM patients were selected for 42 days postpartum to detect the related indexes, it breast feeding was about $47 \%$, mixed feeding was $46 \%$, and pure artificial feeding was $7 \%$. Other pregnant women who took blood tests in the middle pregnancy were not included in this follow-up study because of other complications in the late pregnancy or the loss of medical history due to the absence of treatment in our hospital. The GDM group was divided into 171 patients in the $\mathrm{A} 1$ group and 23 patients 
in the A2 group. All interviewees were followed up for 4 to 6 months. This study was approved by the hospital ethics committee. The participants signed informed consent forms.

\section{Sample analyzes}

After fasting and water deprivation for 12-14 hours, elbow venous blood samples of all enrolled subjects were collected and centrifuged to separate the serum. A portion of serum sample was sent for FPG testing that was preformed using Enzyme electrode kit (Beckman Coulter, Inc. in the United States of America). The rest of serum sample was stored in refrigerator at $-80^{\circ} \mathrm{C}$ for future research.

Dynamic observation of pregnant women in late stage of pregnancy and 42 days after delivery was performed using the same detection method. The data from BMI, HOMAIR, insulin treatment and perinatal outcomes were collected by HIS system. HOMA-IR of Steady State Model was used as an Indicator for Evaluating IR. HOMA-IR = FPG $\times$ FINS/22.5. Finally, the enrolled patients were tested for FINS and RBP4 by enzyme-linked immunosorbent assay (R\&D Systems in the United States of America).

\section{Statistical analyses}

All the data analysis were performed by using SPSS (version 22.0; SPSS Inc, Chicago, IL, USA). All the data were analyzed by descriptive statistics (often detected by Kolmogorov-Smirnov test). The data were expressed as mean $\pm \mathrm{SD}$. The $T$ test was used to compare the variables with normal distribution between the two groups. The ANOVA analysis was used to compare the variables with normal distribution of multiple groups. The rank sum test was used to compare the variables that did not conform to the normal distribution. Two-tailed significance values were given with $\mathrm{P}<0.05$, which was considered to be statistically significant. Correlation relationships analysis was performed by Spearman correlation analysis. When $|\mathrm{r}|<0.5$ and $\mathrm{P}<0.05$, the correlation was low; when $|\mathrm{r}| \geq 0.5$, the correlation was moderate; When $|r| \geq 0.8$, the correlation was high.

ROC curve was used to investigate the sensitivity and specificity of all indicators in GDM diagnosis. ROCcurve analysis with calculation of the area under the curve (AUROC) was performed to calculate the cutoff values, sensitivity, specificity and overall model correctness. AUROC $>0.5$ and $\mathrm{P}<0.05$ were regarded as clinically significant. An AUROC value between 0.5-0.7 indicated low clinical prognostic indicator; AUROC between 0.7-0.9 indicated moderate clinical prognostic indicator; AUROC $>0.9$ indicated high clinical prognostic indicator. Cut-off values were defined by obtaining the best Youden index (Youden index $=$ sensitivity + specificity -1 ).

\section{Results}

\section{Baseline patient characteristics}

In GDM group, the average age was $31.71 \pm 3.63$ years; BMI before pregnancy was $21.74 \pm 2.47 \mathrm{~kg} / \mathrm{m}^{2}$. In normal control group, the average age was $31.00 \pm 3.43$ years; BMI before pregnancy was $21.04 \pm 2.48 \mathrm{~kg} / \mathrm{m}^{2}$. No significant differences in the age, gestational age and pre-pregnancy BMI were observed between groups $(\mathrm{P}>0.05)$.

\section{The comparison of FPG, FINS, HOMA-IR and RBP4 between the two groups of pregnant women at middle pregnancy and late pregnancy}

At middle pregnancy and late stage, levels of FPG, FINS, HOMA-IR and RBP4 were significantly higher in the GDM group compared to the control group $(\mathrm{P}<0.05)$. In addition, in the GDM group, these levels were significantly higher during late stage compared to middle pregnancy $(\mathrm{P}<0.05)$; while, levels of FPG were significantly lower at late stage than those at middle stage $(\mathrm{P}<0.05)$. In control group, there were no differences of FPG, FINS, HOMAIR and RBP4 levels at middle stage of pregnancy and at the late stage $(\mathrm{P}>0.05)$. All results are shown in Table 1 .

\section{Comparison of FINS and RBP4 between pregnant women with GDM, GDM postpartum women and normal pregnant women}

According to Kolmogorov-Smirnov test, FINS had normal distribution in GDM group, normal pregnancy group and GDM after delivery group, and thus ANOVA analysis was utilized. For the normal pregnancy group, no differences were found between middle pregnancy and late stage $(\mathrm{P}>0.05)$; however, significant differences were observed among other groups $(\mathrm{P}<0.05)$. Furthermore, the median levels of FINS in each group were analyzed by box diagram. As shown in Figure 1, the average FINS levels in GDM pregnant women at late stage, GDM pregnant women at middle stage, GDM after delivery, normal pregnant women 
Table 1 The comparison of FPG, FINS, HOMA-IR and RBP4 between the two groups of pregnant women at middle stage pregnancy and late stage

\begin{tabular}{|c|c|c|c|}
\hline Item & GDM group $(n=194)$ & Control group $(n=67)$ & $\mathrm{P}$ \\
\hline \multicolumn{4}{|l|}{ FPG (mmol/L) } \\
\hline Middle stage & $4.82 \pm 0.54$ & $4.37 \pm 0.33$ & $<0.01$ \\
\hline Late stage & $4.69 \pm 0.75$ & $4.31 \pm 0.50$ & $<0.01$ \\
\hline $\mathrm{P}$ & $<0.01$ & 0.40 & - \\
\hline Middle stage & $22.89 \pm 3.73$ & $17.18 \pm 3.06$ & $<0.01$ \\
\hline Late stage & $24.37 \pm 3.42$ & $17.83 \pm 3.84$ & $<0.01$ \\
\hline$P$ & $<0.01$ & 0.05 & - \\
\hline \multicolumn{4}{|l|}{ HOMA-IR } \\
\hline$P$ & $<0.01$ & 0.37 & - \\
\hline \multicolumn{4}{|l|}{ RBP4 ( $\mu \mathrm{g} / \mathrm{mL})$} \\
\hline Middle stage & $41.10 \pm 7.26$ & $30.55 \pm 5.54$ & $<0.01$ \\
\hline Late stage & $43.50 \pm 7.13$ & $31.18 \pm 7.73$ & $<0.01$ \\
\hline$P$ & $<0.01$ & 0.28 & - \\
\hline
\end{tabular}

FPG, fasting plasma glucose; FINS, fasting insulin; RBP4, retinol-binding protein 4; GDM, gestational diabetes mellitus; HOMA-IR, insulin resistance index.

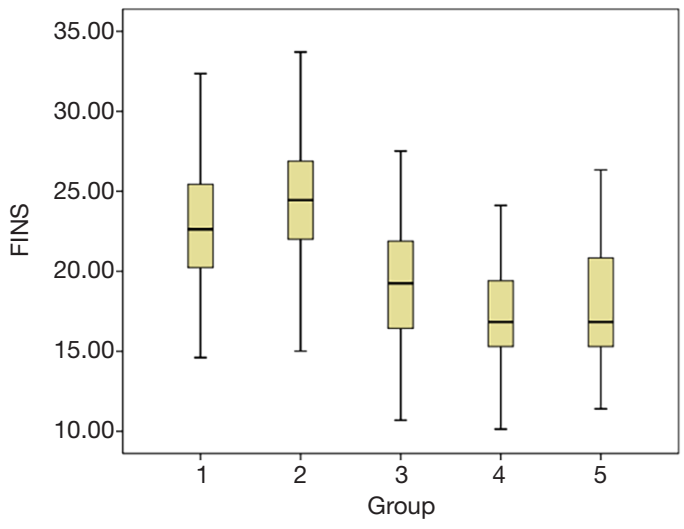

Figure 1 Average FINS levels in GDM pregnant women and normal pregnant women. 1: GDM pregnant women at middle stage, median is $22.63 \mathrm{mU} / \mathrm{L}$. 2: GDM pregnant women at late stage, median is $24.45 \mathrm{mU} / \mathrm{L}$. 3: GDM after delivery, median is $19.25 \mathrm{mU} / \mathrm{L}$. 4: normal pregnant women at middle stage, median is $16.83 \mathrm{mU} / \mathrm{L}$. 5: normal pregnant women at late stage, median is $16.83 \mathrm{mU} / \mathrm{L}$. GDM, gestational diabetes mellitus; FINS, fasting insulin. gradually decreased $(\mathrm{P}<0.01)$.

According to Kolmogorov-Smirnov test, RBP4 in GDM after delivery did not have normal distribution, thus nonparametric test analysis was utilized. The median RBP4 of the GDM after delivery group was $34.10 \mu \mathrm{g} / \mathrm{mL}$. Comparing the median of GDM postpartum group with other groups, significant differences were observed between GDM postpartum group and other groups $(\mathrm{P}<0.05)$. The median RBP4 value of GDM after delivery was lower than the value of GDM at late stage and middle stage. In addition, the median RBP4 value of GDM after delivery was higher compared to normal pregnancy at late stage and middle stage (Figure 2).

\section{Relationship between serum RBP4 concentration and other parameters}

Serum RBP4 in pregnant women with GDM was highly correlated with FINS and moderately correlated with HOMA-IR at the middle pregnancy, it was moderately 


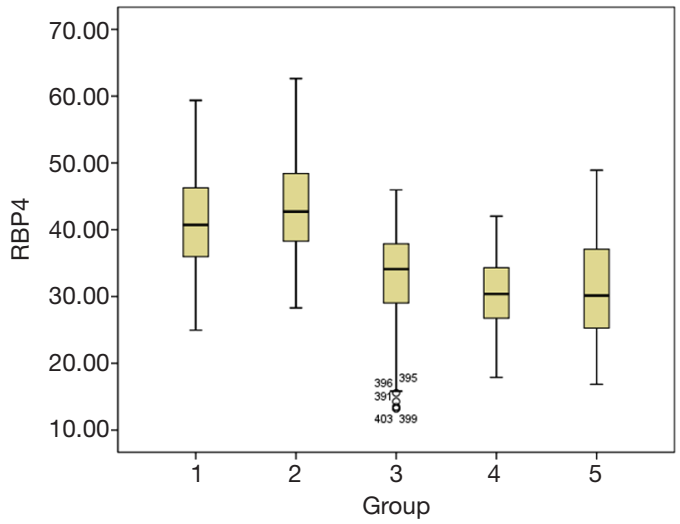

Figure 2 Average RBP4 levels in GDM pregnant women and normal pregnant women. 1: GDM pregnant women at middle stage, median is $40.72 \mu \mathrm{g} / \mathrm{mL}$. 2: GDM pregnant women at late stage, median is $42.70 \mu \mathrm{g} / \mathrm{mL}$. 3: GDM after delivery, median is $34.10 \mu \mathrm{g} / \mathrm{mL}$. 4: normal pregnant women at middle stage, median is $30.38 \mu \mathrm{g} / \mathrm{mL}$. 5 : normal pregnant women at late stage, median is $30.14 \mu \mathrm{g} / \mathrm{mL}$. GDM, gestational diabetes mellitus; RBP4, retinolbinding protein 4 . correlated with FINS and low correlation with HOMA-IR at late pregnancy. In the control group, serum RBP4 was highly correlated with FINS and HOMA-IR at the middle pregnancy, it was highly correlated with FINS and HOMAIR at late pregnancy. Serum RBP4 in GDM postpartum group was highly positively correlated with FINS, there was a moderate positive correlation with HOMA-IR. The levels of serum RBP4 in these five groups were not correlated with age, FPG, blood glucose at $1 \mathrm{~h}$ and $2 \mathrm{~h}$ after sugar and BMI $(\mathrm{P}>0.05)$ (Table 2).

\section{GDM diagnostic receiver operating characteristic curve}

Based on the ROC curve, the optimal cut-off value of FINS at middle pregnancy as an indicator for GDM diagnosis was projected to be $20.22 \mathrm{mU} / \mathrm{L}$, with sensitivity of $75.3 \%$ and a specificity of $85.1 \%$, and an AUC area of 0.88 (95\% CI: $0.84-0.92 ; \mathrm{P}<0.01)$. The optimal cut-off value of HOMAIR at middle pregnancy as an indicator for GDM diagnosis was projected to be 4.06 , with sensitivity of $85.1 \%$ and a

Table 2 Analysis of the correlation between serum RBP4 and FPG, OGTT, HOMA-IR and FINS in five groups of women

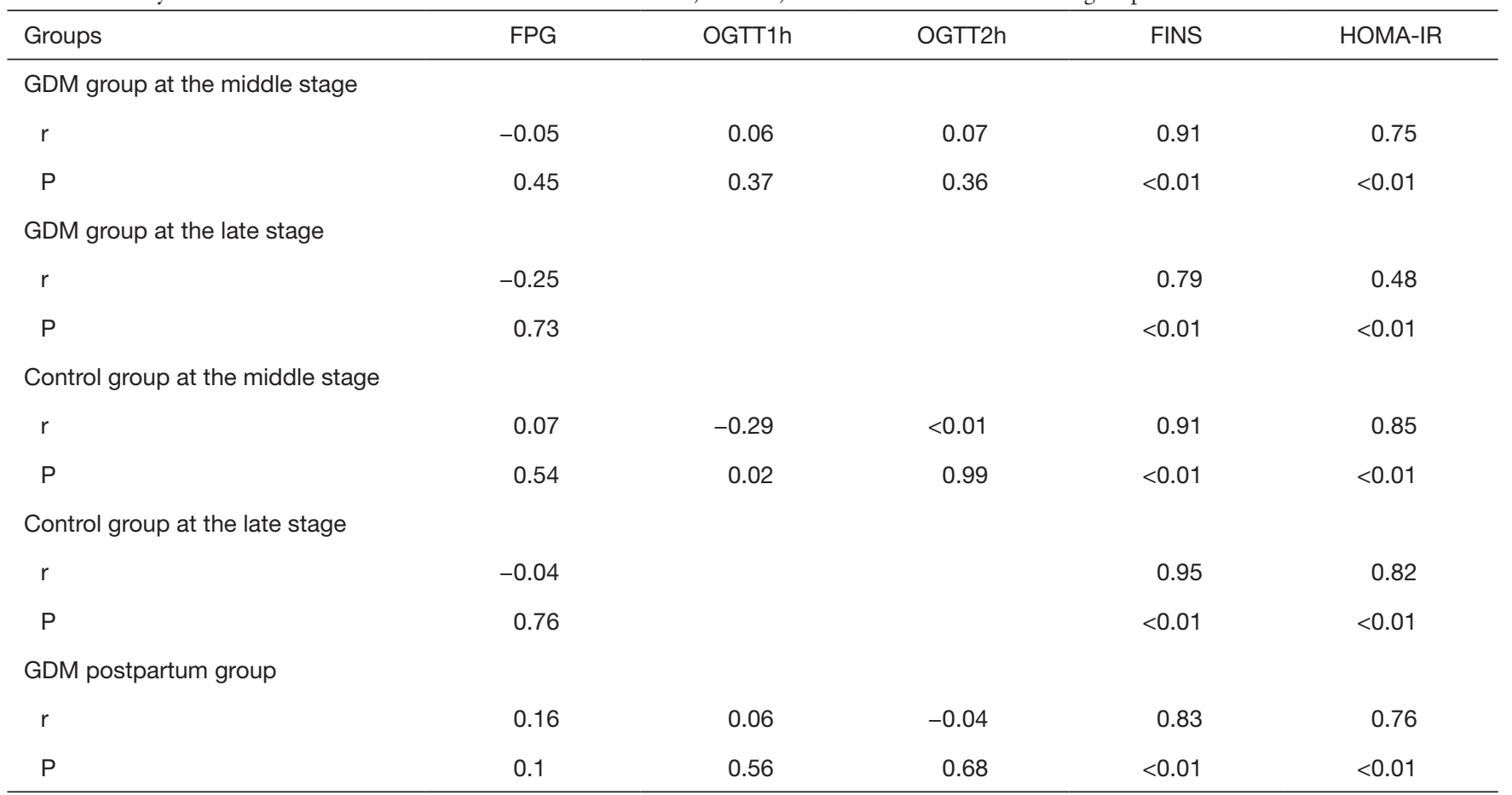

FPG, fasting plasma glucose; FINS, fasting insulin; RBP4, retinol-binding protein 4; GDM, gestational diabetes mellitus; HOMA-IR, insulin resistance index. 
specificity of $86.6 \%$, and an AUC area of 0.92 (95\% CI: $0.88-0.95 ; \mathrm{P}<0.01)$. The optimal cut-off value of RBP4 at middle pregnancy in GDM diagnosis was $34.84 \mu \mathrm{g} / \mathrm{mL}$, with sensitivity and specificity of $79.4 \%$ and $79.1 \%$, respectively, and an AUC area of 0.87 (95\% CI: 0.83-0.92; $\mathrm{P}<0.01)$. Other meaningless indicators were not included in the analysis. The results are presented in Figure 3 and Table 3.

\section{Differences between A1GDM and A2GDM group}

OGTT0h, $2 \mathrm{~h}$, middle stage HOMA-IR and late stage pregnancy FPG were all higher in A2GDM group compared to A1GDM group, and the difference was statistically significant $(\mathrm{P}<0.05)$. In addition, no significant differences between the two groups were found for age, BMI during pregnancy, OGTT1h, FINS and RBP4 during pregnancy, and HOMA-IR at late pregnancy $(\mathrm{P}>0.05)$.

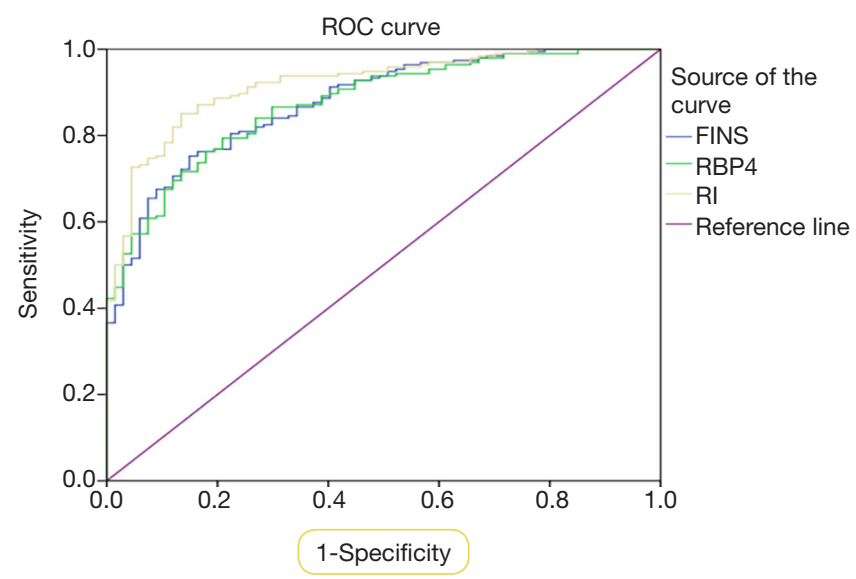

Figure 3 GDM diagnostic ROC curve of FINS, HOMA-IR, RBP4 at middle pregnancy. GDM, gestational diabetes mellitus; FINS, fasting insulin; RBP4, retinol-binding protein 4; HOMA$\mathrm{IR}$, insulin resistance index.
Results are shown in Table 4.

\section{Discussion}

Previous research has found that the loads of various organs are positively correlated with the gestational age during pregnancy. During this period, secretion of antagonizing insulin increases, which in turn may lead to low sensitivity of surrounding tissue to insulin. When insulin secretion is insufficient, GDM may occur (18). Serum RBP4, a protein that regulates the transportation of retinol and is mainly secreted by the liver and adipose tissue, has an important role in insulin resistance (7). So far, several studies have suggested an association between RBP4 and GDM. In this study, serum RBP4 from middle stage to late stage and postpartum were collected from GDM pregnant women and normal control pregnant women, and their relationship with various glucose metabolism related indicators was analyzed. Briefly, the correlation and predictive value with GDM were found. By comparing the relative indexes of different grades (A1 and A2) in pregnant women with GDM, we aimed to identify the high-risk factors of A2GDM.

In our study, levels of RBP4, FINS and HOMA-IR in GDM were significantly higher compared to the healthy controls regardless of the pregnancy stage. Consistent with many previous study results $(19,20), \mathrm{RBP} 4$ were associated with IR and GDM. Yet, some researchers showed different results (15). In addition, we found that with increasing gestational age, levels of RBP4, FINS and HOMA-IR statistically increased at late stage compared with those at middle pregnancy in GDM group. In the control group, levels of RBP4, FINS and HOMA-IR during middle stage and late stage were similar. We suppose that with the increase in gestational age, the sensitivity of surrounding tissue to insulin decreases, while insulin resistance increases.

Table 3 ROC-curve analysis results of FINS, HOMA-IR and RBP4 in GDM diagnosis

\begin{tabular}{lcccccc}
\hline At middle pregnancy & AUC & $95 \% \mathrm{Cl}$ & P value & Optimal cut-off value & Sensitivity (\%) & specificity (\%) \\
\hline FINS $(\mathrm{mU} / \mathrm{L})$ & 0.88 & $0.84-0.92$ & $<0.01$ & 20.22 & 75.3 & 85.1 \\
HOMA-IR & 0.92 & $0.88-0.95$ & $<0.01$ & 4.06 & 34.1 & 86.6 \\
RBP4 $(\mu \mathrm{g} / \mathrm{mL})$ & 0.87 & $0.83-0.92$ & $<0.01$ & 34.84 & 79.1 \\
\hline
\end{tabular}

FINS, fasting insulin; RBP4, retinol-binding protein 4; GDM, gestational diabetes mellitus; HOMA-IR, insulin resistance index. 
Table 4 Analysis of related high-risk factors in A1GDM group and A2GDM group

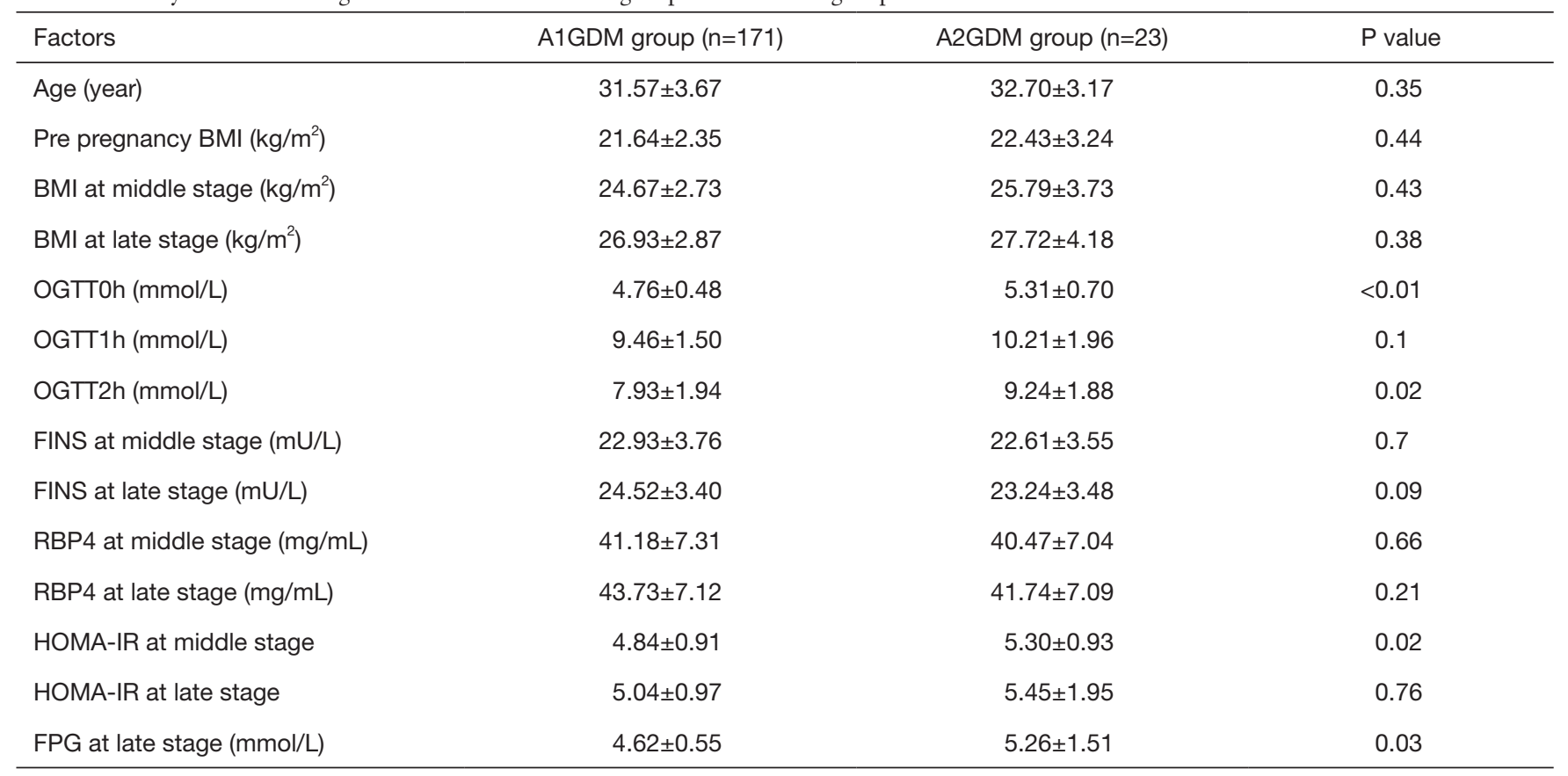

BMI, body mass index; FPG, fasting plasma glucose; FINS, fasting insulin; RBP4, retinol-binding protein 4; GDM, gestational diabetes mellitus; HOMA-IR, insulin resistance index.

RBP4 is closely related to insulin resistance, so the RBP4 value of GDM pregnant women is also significantly increased. In normal pregnant women, the decrease in insulin sensitivity is adapted to IR; therefore, RBP4 is not as significantly elevated as in GDM pregnant women.

Our study indicated that FPG at late stage was lower than that at middle pregnancy in GDM. FPG decrease may result from blood glucose controlled by dietary treatment in most enrolled patients after diagnosis of GDM. Although FPG was decreased in GDM, the levels of RBP4, FINS and HOMA-IR were all increased. We assume that FINS and RBP4 levels were not affected by blood glucose, i.e., FINS and RBP4 could reflect the insulin sensitive status and development in GDM.

Our study showed that the FINS and RBP4 levels of healthy controls 42 days after delivery were significantly lower than those of pregnant women with GDM. Nevertheless, levels of FINS and RBP4 in GDM patients after delivery were statistically higher compared to the control group at middle stage and late stage. Chan et al. (13) have reported that RBP4 levels at 24-28 weeks of gestational age in GDM patients were higher compared to healthy controls. In his study, RBP4 levels in GDM patient were associated with BMI and they rapidly decreased after delivery, while this phenomenon was not observed in healthy controls. Based on our data, we presumed that the insulin resistance decrease is positively correlated with FINS and RBP4 levels, which also tends to decrease. FINS and RBP4 levels, which are the most sensitive indicators of insulin resistance at middle pregnancy and late pregnancy in GDM were higher than those in normal pregnancy, which suggested that insulin resistance had little effect on normal pregnant women. Yet, in GDM, high levels of insulin resistance may lead to a greater impact on the insulin receptor. The risk of developing type 2 diabetes after delivery occurs when the pancreas cannot make enough insulin to keep blood sugar at normal levels. Jang and colleagues (21) have shown that $10-15 \%$ of postpartum GDM patients tend to develop type 2 diabetes mellitus in the near term after delivery.

In this study, we found that serum RBP4 level was highly correlated with FINS and HOMA-IR at middle stage pregnancy and late pregnancy in GDM, normal pregnancy and GDM postpartum group $(\mathrm{P}<0.05, \mathrm{r}>0.50)$. FINS and HOMA-IR reflect the state of insulin resistance, indicating that serum RBP4 is associated with insulin resistance in GDM, normal pregnant women and GDM postpartum group. No relationship was found in serum RBP4 and FPG 
between two groups. We presume that RBP4 reflects longterm IR status in pregnancy, which is unrelated to FPG. Previous studies were not consistent with reference to the relationship between RBP4, FINS and HOMA-IR. Du et al. (22) have shown that RBP4 is highly correlated with HOMA-IR in normal group, while there was no correlation in the GDM group; nevertheless, the number of included patients was small (38 cases were included in the GDM group). In the present study, there was no relationship among age, $\mathrm{BMI}$ and $\mathrm{RBP} 4(\mathrm{P}>0.05)$. One possible reason for this is that BMI of GDM group and BMI of normal control group were similar in order to eliminate the effects of obesity and age on RBP4 value.

Furthermore, we found that FINS, HOMA-IR and RBP4 levels observed during middle stage can be used as predictors of GDM diagnosis. The optimal cut-off value of RBP4 at middle pregnancy was $34.84 \mu \mathrm{g} / \mathrm{mL}$ with sensitivity of $79.4 \%$ and specificity of $79.1 \%$. Maghbooli and his team (23) have discovered that RBP4 concentration is significantly higher in GDM patients compared to normal glucose tolerance in pregnant women. In his study, we found that RBP4 $\geq 42 \mathrm{ug} / \mathrm{mL}$ could be used for GDM diagnosis, and that RBP4 concentration was a risk factor. Du et al. (24) examined 827 subjects and found that RBP4 $\geq 23.4 \mu \mathrm{g} / \mathrm{mL}$ could be used to predict GDM. The results of RBP4 in this study are different from those of Maghbooli and Du, considering the different gestational weeks or races of the subjects.

Levels of OGTT0h and $2 \mathrm{~h}$ in A2GDM group were significantly increased compared to A1GDM group. In view of the results of this study, the author has found fewer relevant reports. Higher levels of OGTT0h and $2 \mathrm{~h}$ experiment at 24-28 weeks of gestation may indicate more severe GDM status. Due to continuously increasing levels of insulin resistance in GDM, blood glucose become worse even if more insulin is secreted. Therefore, OGTT value becomes high. The worse the insulin regulation function of GDM pregnant women is, the more severe the condition will be at late pregnancy. According to our study results, the most sensitive indicators for A2GDM were OGTT0h and $2 \mathrm{~h}$ levels. In clinical practice, when OGTT0h and $2 \mathrm{~h}$ levels are high at antenatal care, clinicians should be more cautious to closely monitor blood glucose in GDM. When blood glucose cannot be controlled with diet and physical activity, insulin treatment should be used in order to reduce complications in GDM.

At late stage, higher levels of FPG were observed in the A2GDM group compared to A1GDM group. The value was consistent with the clinical practice. The simple diet regulation was not enough to control the blood glucose, thus the insulin treatment was needed. Therefore, the FPG level of A2GDM group is generally high. HOMA-IR in A2GDM group were significantly higher than HOMAIR in A1GDM group at middle stage. However, there was no difference between A1GDM and A2GDM groups in HOMA-IR at late stage, which might be related to the small number of cases in the group, which should be addressed by future studies.

There was no difference in the value of RBP4 between A1GDM group and A2GDM group, which may be due to following reasons: (I) the number of cases in this group was small, thus could not accurately reflect the predictive value of RBP4; (II) RBP4 levels just reflect the current insulin resistance status, and cannot precisely predict the severity of long-term illness. Arrigo Fruscalzo (25) has reported that the level of RBP4 in A2GDM group is lower than that in A1GDM group, but he also reported that the number of analyzed cases in his study was too small (only 11 cases in A2GDM group and 21 cases in A1GDM group).

This study is subject to certain limitations: (I) postpartum follow-up of GDM pregnant women is difficult, the number of cases is small, and healthy pregnant women are not included in the postpartum study, resulting in incomplete postpartum data analysis; (II) the information of diet and physical activity of pregnant women has not been studied in detail, and the effects of these confounding factors on RBP4 and FINS metabolite characteristics have not been evaluated; (III) the number of A2GDM cases was small, and the specific dose of insulin was not included in the study.

\section{Conclusions}

The major findings of the current study are the following: the occurrence of RBP4 and IR is closely related to the pathogenesis of GDM. In GDM pregnant patients, RBP4, FINS and HOMA-IR levels are positively correlated with gestational age, which further indicates that the insulin resistance in these patients has been aggravated, and the sensitivity is not affected by immediate fasting blood glucose, which may reflect the development of GDM. Although the postpartum insulin resistance of GDM in pregnant women significantly decreased, RBP4 and FINS were still higher compared to normal pregnant women group. This indicated that RBP4 may reflect the impaired insulin function of GDM in pregnant women, which is still difficult to recover in the short term after birth, where even 
the damaged state can lead to future risks of type 2 diabetes. In addition, during middle stage, FINS $\geq 20 \mathrm{mU} / \mathrm{L}$, RBP4 $\geq 35 \mathrm{mg} / \mathrm{mL}$ has a certain significance for the diagnosis of GDM. Compared with OGTT1h, higher OGTT0h and $2 \mathrm{~h}$ levels at middle pregnancy lead to the higher risk of A2GDM at late stage, which is something that clinicians should pay more attention to during early treatment.

\section{Acknowledgments}

Thank teachers and doctor of Obstetrics, Institute of Maternal and Child Research, Department of Science and Education and Laboratory of our hospital for their kindly help in designing the project, consulting, collecting specimens and so on. In particular, thank Dr. Li Chao of National Cancer Center/Cancer Hospital \& Shenzhen Hospital, Chinese Academy of Medical Sciences and Peking Union Medical College for revision of this manuscript.

Funding: The Shenzhen Science and Technology Innovation Commission supported this study (grant number: JCYJ20130402094702483).

\section{Footnote}

Conflicts of Interest: The authors have no conflicts of interest to declare.

Ethical Statement: The authors are accountable for all aspects of the work in ensuring that questions related to the accuracy or integrity of any part of the work are appropriately investigated and resolved. This study was approved by the hospital ethics committee. The participants signed informed consent forms.

\section{References}

1. Buchanan TA, Xiang A, Kjos SL, et al: What is gestational diabetes? Diabetes Care 2007;30:S105-11.

2. American Diabetes Association. Diagnosis and classification 0f diabetes mellitus. Diabetes Care 2011;40:62-9.

3. Tutino GE, Tam WH, Yang X, et al. Diabetes and pregnancy: perspectives from Asia. Diabet Med 2014;31:302-8.

4. Rosenbloom JI, Blanchard MH. Compliance with Postpartum Diabetes Screening Recommendations for Patients with Gestational Diabetes. J Womens Health (Larchmt) 2018;27:498-502.
5. Tehrani FR, Hashemi S, Hasheminia M, et al. Follow-up of women with gestational diabetes in the Tehran Lipid and Glucose Study (TLGS): a population-based cohort study. J Obstet Gynaecol Res 2012;38:698-704.

6. Gunderson EP, Hedderson MM, Chiang V, et al. Lactation intensity and postpartum maternal glucose tolerance and insulin resistance in women with recent GDM: the SWIFT cohort. Diabetes Care 2012;35:50-6.

7. Yang Q, Graham TE, Mody N, et al. Serum retinol binding protein 4 contributes to insulin resistance in obesity and type 2 diabetes. Nature 2005;436:356-62 .

8. Majerczyk M, Olszanecka-Glinianowicz M, PuzianowskaKuźnicka M, et al. Retinol-binding protein 4 (RBP4) as the causative factor and marker of vascular injury related to insulin resistance. Postepy Hig Med Dosw (Online) 2016;70:1267-75.

9. Chen Y, Lv P, Du M, et al. Increased retinol-free RBP4 contributes to insulin resistance in gestational diabetes mellitus. Arch Gynecol Obstet 2017;296:53-61.

10. Wang MN, Han YB, Li Q, et a1. Higher Serum Retinol Binding Protein 4 May be a Predictor of Weak Metabolic Control in Chinese Patients with Type 2 Diabetes Mellitus. J Int Med Res 2012;40:1317-24.

11. Feng $\mathrm{S}, \mathrm{Zhu} \mathrm{Y}$, Yan $\mathrm{C}$, et al. Retinol binding protein 4 correlates with and is an early predictor of carotid atherosclerosis in type 2 diabetes mellitus patients. J Biomed Res 2015;29:451-5.

12. Abetew DF, Qiu C, Fida NG, et al. Association of retinol binding protein 4 with risk of gestational diabetes. Diabetes Res Clin Pract 2013;99:48-53.

13. Chan TF, Chen HS, Chen YC, et al. Increased serum retinol-binding protein 4 concentrations in women with gestational diabetes mellitus. Reprod Sci 2007;14:169-74.

14. Tepper BJ, Kim YK, Shete V, et al. Serum retinolbinding protein 4 (RBP4) and retinol in a cohort of borderline obese women with and without gestational diabetes. Clin Biochem 2010;43:320-3.

15. Khovidhunkit W, Pruksakorn P, Plengpanich W, Tharavanij T. Retinol-binding protein 4 is not associated with insulin resistance in pregnancy. Metabolism 2012;61:65-9.

16. Kim YL, Kim TK, Cheong ES. Relation of absolute or relative adiposity to insulin resistance, retinol binding protein- 4 , leptin, and adiponectin in type 2 diabetes. Diabetes Metab J 2012;36:415-21.

17. Hod M, Kapur A, Sacks DA, et al. The International Federation of Gynecology and Obstetrics ( FIGO) Initiative on gestational diabetes mellitus: A pragmatic 
guide for diagnosis, management, and care. Int J Gynaecol Obstet 2015;131:173-211.

18. Yilmaz O, Kucu KM, Ilgin A, et al. Assessment of insulin sensitivity/resistance and their relations with leptin concentrations and anthropometric measures in a pregnant population with and without gestational diabetes mellitus. J Diabetes Complications 2010;24:109-14.

19. Kuzmicki M, Telejko B, Wawrusiewicz-kurylonek N, et al.Retinol-binding protein 4 in adipose and placental tissue of women with gestational diabetes. Gynecol Endocrinol 2011;27:1065-9.

20. Gursoy AY, Aynaoglu G, Caglar GS, et al. Early second trimester retinol-binding protein-4 values in cases with or without gestational diabetes mellitus risk factors: a crosssectional study. J Obstet Gynaecol Res 2015;41:55-61.

21. Jang HC. Gestational diabetes in Korea: incidence and risk factors of diabetes in women with previous gestational diabetes. Diabetes Metab J 2011;35:1-7.

Cite this article as: Du X, Dong Y, Xiao L, Liu GH, Qin $\mathrm{W}, \mathrm{Yu} \mathrm{H}$. Association between retinol-binding protein 4 concentrations and gestational diabetes mellitus (A1GDM and A2GDM) in different pregnancy and postpartum periods. Ann Transl Med 2019;7(18):479. doi: 10.21037/atm.2019.08.45
22. Du M, Wang B, Liang Z, et al. The Relationship between Retinol-Binding Protein 4 Concentrations and Gestational Diabetes Mellitus in Chinese Women. Gynecol Obstet Invest 2015;81:174-80.

23. Maghbooli Z, Hossein-Nezhad A, Mirzaei K, et al. Association between retinol-binding protein 4 concentrations and gestational diabetes mellitus and risk of developing metabolic syndrome after pregnancy. Reprod Sci 2010;17:196-201.

24. Du C, Kong F. A Prospective Study of Maternal Plasma Concentrations of Retinol-Binding Protein 4 and Risk of Gestational Diabetes Mellitus. Ann Nutr Metab 2019;74:1-8.

25. Fruscalzo A, Londero AP, Driul L, et al. First trimester concentrations of the TTR-RBP4-retinol complex components as early markers of insulin-treated gestational diabetes mellitus. Clin Chem Lab Med 2015;53:1643-51. 